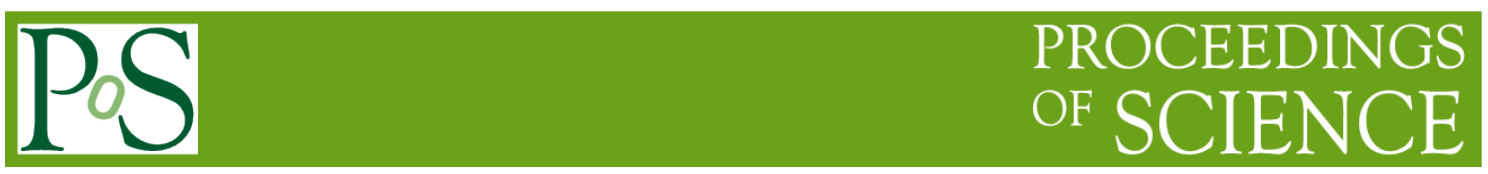

\title{
Time dependent Geomagnetic Cutoff estimation along the ISS orbit
}

E. Fiandrini ${ }^{\text {ab, }}$, M. Duranti ${ }^{\text {ab,2 }}$, B. Bertucci ${ }^{\text {ab }}$, M. Boschini ${ }^{\mathrm{d}}$, M. Crispoltoni ${ }^{\mathrm{ab}}$, S. Della Torre ${ }^{\mathrm{d}}$, F. Donnini ${ }^{\text {ab }}$, D. D'Urso ${ }^{\text {ac }}$, V. Formato ${ }^{\mathrm{a}}$, M. Gervasi ${ }^{\mathrm{de}}$, D. Grandi ${ }^{\mathrm{d}}$, M. Graziani ${ }^{\mathrm{ab}}$, G. La Vacca ${ }^{\mathrm{d}}$, F. Nozzoli $^{\text {ac }}$, S. Pensotti ${ }^{\text {de }}$, C. Pizzolotto ${ }^{\text {ac }}$, P.G. Rancoita ${ }^{\mathrm{d}}$, D. Rozza ${ }^{\text {de }}$, M. Tacconi ${ }^{\text {d }}$, V. Vitale ${ }^{\text {ac }}$, M. Zannonide.

In this contribution we present the calculation of a realistic time dependent geomagnetic cutoff along the International Space Station orbit, at about $400 \mathrm{~km}$ above the Earth's surface with an inclination of $51.6^{\circ}$. For this work, based on the analysis of data collected by the AMS02 experiment during the first year of operation, the Tsyganenko 05 and IGRF models have been employed, including the temporal variation of the external component of the geomagnetic field due to the solar activity. The evaluation of the cutoff has been performed every 30 seconds along the ISS orbit by means of a back-tracing technique of 1500 random trajectories simulated in the field of view of the AMS-02 experiment at any given ISS location. The technique and its results will be discussed, as well as the relevance of this study to distinguish galactic cosmic rays from trapped secondary components in the geomagnetic field.

${ }^{a}$ INFN Perugia, I-06124 Perugia, Italy

${ }^{b}$ Università di Perugia, I-06124, Perugia, Italy

${ }^{c}$ ASI Science Data Center, I-00133 Roma, Italy

${ }^{\mathrm{d} I N F N ~ S e z i o n e ~ d i ~ M i l a n o ~ B i c o c c a, ~ I-20126 ~ M i l a n o, ~ I t a l y ~}$

eUniversità di Milano Bicocca, I-20126 Milano, Italy

The 34th International Cosmic Ray Conference

30 July- 6 August, 2015

The Hague, The Netherlands

${ }^{1}$ Corresponding author, University and INFN Perugia,Via Pascoli, Italy

E-mail: emanuele.fiandriniepg. infn.it 


\section{Introduction}

The charged galactic cosmic rays (CR) arrive at the top of atmosphere with an isotropic distribution. The presence of the geomagnetic field breaks the isotropy at low energies $(O(10 \mathrm{GeV}))$, since it deviates the particles trajectories, such that only particles with enough magnetic rigidity $\mathrm{R}=\mathrm{p} / \mathrm{Ze}$ can reach from in finity a given position from a given direction, as shown in Fig. 1. This means that there is a screen effect for particles with a rigidity $R$ below a threshold, called cut-off rigidity $R_{c}$, which depends on the geomagnetic position, arrival direction with respect to the local $\mathrm{B}$ field and the time. Formally, the cutoff rigidity can be calculated analitically only in dipolar field [1,2]. However, the real geomagnetic field is much more complex than a simple dipole, as seen in Fig. 3 and Fig. 4 and some trajectories, that would be allowed, become forbidden due to the presence of the solid Earth that Fig. 1. Allowed and forbidden trajectories in a intercepts them. The best way to take

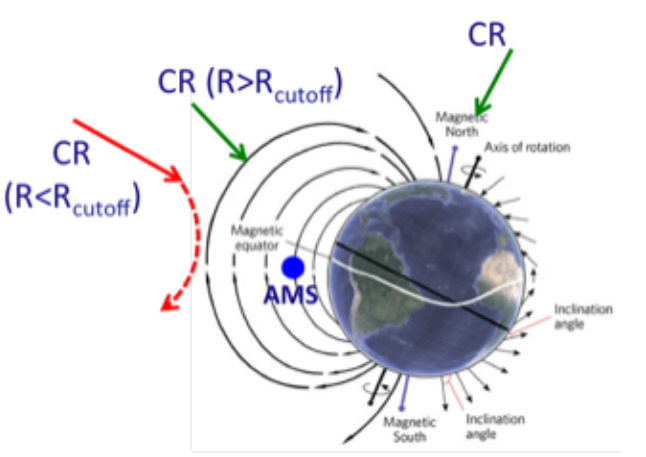
dipolar field into account these effects is to use a back-tracing tecnique in the geomagnetic field by integrating the particle motion from a known position and direction in a realistic field model $[12,13,14]$. The cut-off rigidity has to be used as an ordering parameter for CR measurements, since it is mandatory to disentagle the galactic cosmic ray population from the trapped particles underneath the radiation belts which are measured at the altitudes of Low Earth Orbits in order to properly determine the fluxes, as shown in Fig. 2. The low energy population is made of particle produced in the atmosphere and trapped in the geomagnetic field (e.g. the Cosmic Ray Albedo Neutron Decay, CRAND), while the high energy component is of galactic origin. As can be seen, at every position can be de fined a minimum rigidity

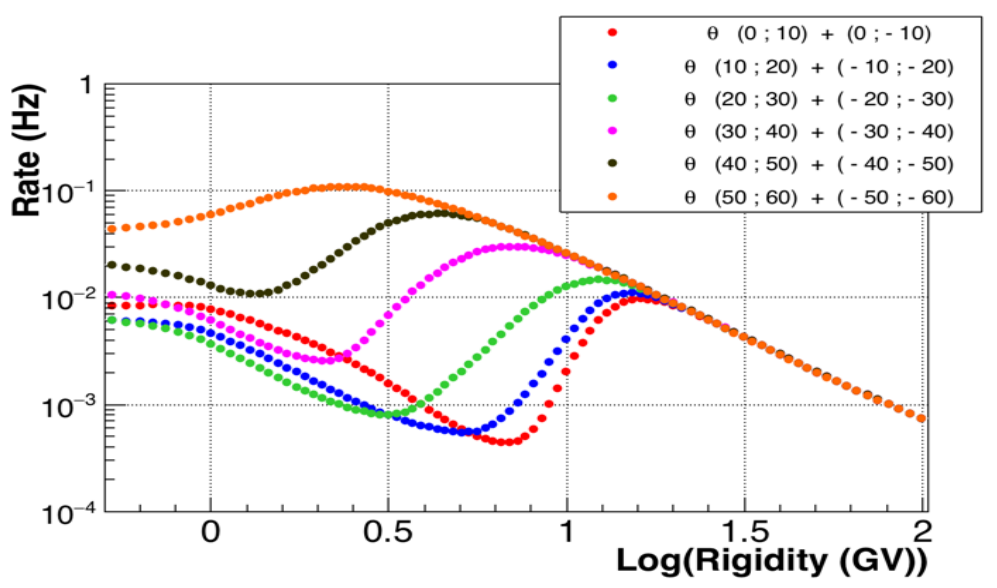

Fig.2. Counting rate as a function of $\mathrm{R}$ at different magnetic latitudes. Two distinct populations are clearly seen, separated by the cutoff rigidity 
which separates the two components. Below this rigidity a particle from in finity can not reach the position and a trapped particle can not escape the field. For an optimal separation, it is necessary to know at every time the value of the geomagnetic cutoff. Particularly interesting is to calculate the cut-off rigidities along the orbit of the International Space Station (ISS) because it hosts important experiments, as the AMS02 spectrometer and other experiments will be installed, as CALET and ISS-CREAM.

In this work, we present the calculated cut-off rigidity along the ISS for a period of one year, beginning from July $1^{\text {st }} 2011$. The cut-off was calculated every 30 seconds, using the position and pointing data provided by the AMS02 apparatus [10]. The ISS orbit is almost circular at an altitude of about $400 \mathrm{Km}$ with an inclination of $51.6^{\circ}$ with respect to the equator. The counting rates registered by AMS02 at different latitudes are shown in Fig. 2, where the two populations, partially overlapping, are clearly visible.

The back-tracing tecnique used here is based on a 4th order Runge-Kutta integration with the "turning angles" method to optimize the integration step: it is chosen such that the maximal deflection in the step is below a fixed value of $3 \mathrm{mrad}$. It was adapted from previous works $[16,17,18]$ to make it running much faster since the goal is to provide the upper and lower cutoffs along the ISS orbit for a very long period of time.

\section{Geomagnetic field models}

Crucial for the calculation of a realistic cut-off is the choice of the geomagnetic field model. The magnetosphere is a dynamic system that changes both on short time scale, due to to interactions with the solar wind, and on longer time scale due to the secular variations of the field. The cutoff rigidity depends on the magnetic field, so its choice is

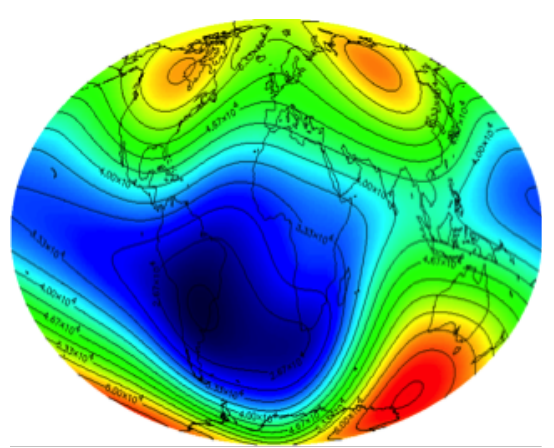

Fig. 3: Map of internal IGRF field at the altitude of ISS orbit

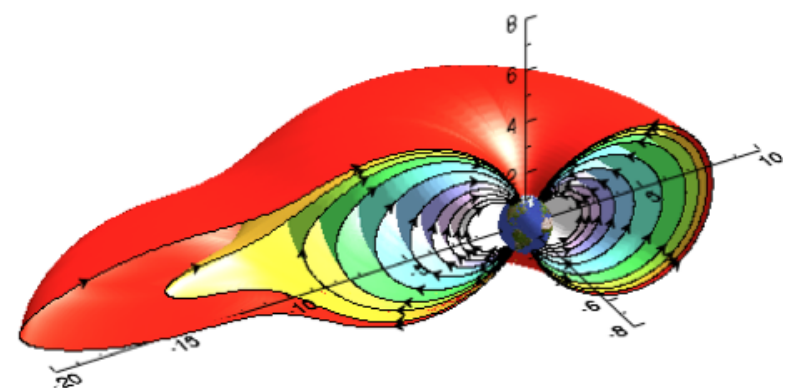

Fig. 4: Semi-empirical model of Tsyganenko05 external field [9]. Sun is to the right, along $\mathrm{x}$ axis. Units are Earth radii $\mathrm{Re}$.

crucial. The Earth's magnetic field is usually represented as the sum of two contributions, one due the Earth's dynamo, referred as internal field, the other due to the complex system of currents circulating in the magnetosphere (e.g. ring current), usually referred as external field. The internal field causes secular variations (e.g. internal field decreased by $39 \%$ during last 400 years [9]), while the external one is strongly influenced by solar activity and is particularly important during the geomagnetic distubances, as storms, flares, coronal mass ejections, but not negligible during quiet periods, even for just night and day cycle. For this work, the field models available were 
IGRF10 [3], valid until 2015, for the internal component, and Tsyganenko05, 98, 89, 86 [4],[7], Paraboloid [10], Matsev [6] and Olson\&Pfitzer [5] for the external field. More references on field models can be found in [13]. The software package used to evaluate the magnetic field is UNILIB [15]. The external field parameters $\left(\mathrm{Ds}_{t}, \mathrm{~B}_{\mathrm{imf}}, \mathrm{V}_{\mathrm{sw}}, \mathrm{P}_{\mathrm{sw}}\right.$, ...) were updated every $300 \mathrm{sec}$, while planetary activity index $\mathrm{K}_{\mathrm{p}}$ every 3 hours, by using the available data from NASA databases, so that the time variations of field can be taken into account according to the space weather conditions. The particle trajectories were traced back from a given position until one of the following conditions occurs: 1) path length reaches 1500 Earth'radii $\left(R_{e}\right), 2$ ) intercepts the atmosphere at $20 \mathrm{~km}$ above sea level. 3) a distance of $12 \mathrm{R}_{\mathrm{e}}$ from the field center is reached, 4) trajectory intercepts the magnetopause. Conditions 1) and 2) define trajectories not reaching infinity, therefore "trapped" in the geomagnetic field, conditions 3) and 4) de fine trajectories that escape Earth's magnetosphere and reach in finity, de fined as "primary" trajectories.

\section{Transition Function}

In principle, at a given position the cut-off rigidity depends on the arrival direction (e.g. the east-west asymmetry), so that for any position a whole set of cutoffs should be given. This is unpractical and not needed for most of the applications. At any position, it is more practical to calculate a cutoff rigidity averaged over the the arrival directions within a cone of given aperture. For this purpose, at every position of the ISS, 1500 trajectories were generated with: i) random directions in cone with aperture $40^{\circ}$ with respect to AMS02 zenith (inclined of $12^{\circ}$ with respect to ISS), ii) random rigidities in a loose range $\left(\mathrm{R}_{\min }, \mathrm{R}_{\max }\right)$, position dependent, around the expected cutoff, to optimize the back-tracing and to avoid to trace trajectories that are certainly primary or

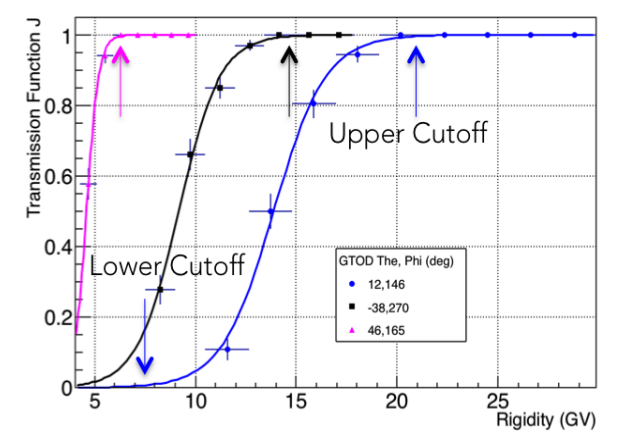

Fig. 5. Transition function averaged over the arrival directions as a function of rigidity for three different locations within the geomagnetic field, at the altitude of ISS.

secondaries. The rigidity window was determined with a dedicated set of back-traced trajectories at a fixed arbitrary time, iii) for each trajectory, the transmission index $J_{\text {trans }}$ was defined by using the back tracing. A trajectory intercepting the atmosphere or reaching the max path length was given $\mathrm{J}_{\text {trans }}=0$ ("trapped"), while $\mathrm{J}_{\text {trans }}=1$ ("primary") was given to trajectories reaching the max distance or intercepting the magnetopause, iv) for every rigidity, $\mathrm{J}_{\text {trans }}$ was averaged over the arrival directions, as shown in fig. 5. The transition from trapped to primary is not sharp, as is clearly seen in 
fig.5. This is due both to the averaging over the arrival directions and to the Earth's penumbra and field irregularities. It is possible, however, to de fine two rigidities that characterize trapped and primary trajectories:

$\checkmark$ an upper cutoff as the minimum rigidity such that all the trajectories are primary_

$$
\left(\mathrm{J}_{\text {trans }}=1\right)
$$

$\checkmark$ a lower cutoff as the maximum rigidity such that all the trajectories are trapped $\left(\mathrm{J}_{\text {trans }}=0\right)$.

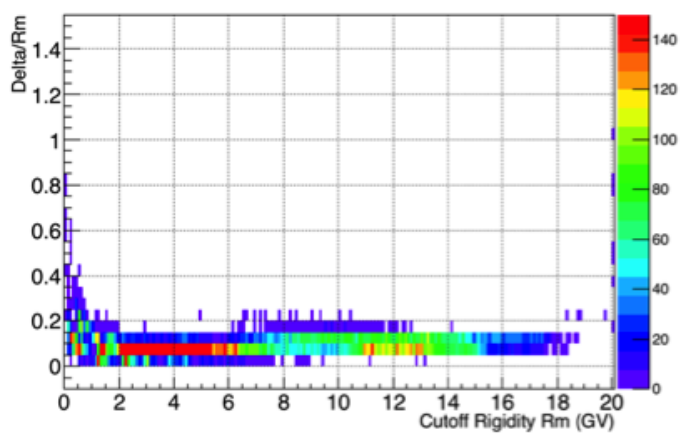

Fig. 6. Ratio of $\delta / R_{m}$ as a function of cutoff rigidity $R_{m}$.

$\mathrm{J}_{\text {trans }}$ is well described by a Fermi-Dirac type function $\mathrm{J}(\mathrm{R})=1 /\left[1+\exp \left(\left(\mathrm{R}-\mathrm{R}_{\mathrm{m}}\right) / \boldsymbol{\delta}\right)\right]$ where $\mathrm{R}_{\mathrm{m}}$ is the rigidity for which $50 \%$ of trajectories are primaries and $\delta$ is the width of the penumbra region. The ratio $\delta / R_{m}$ is shown in fig. 6 . It is worth to note that this ratio does not depend on the cutoff rigidity, that it is independent of position, time and defines the penumbra width, which scales linearly with cutoff rigidity. Another approach by the same group, where real AMS02 data are traced-back to calculate the cutoff is presented in the same proceedings [14].

\section{Geomagnetic Cutoff}

In this work we have calculated the upper and lower cutoff along the ISS orbit over a period of one year, beginning form July 1st 2011 every 30 seconds for positively and

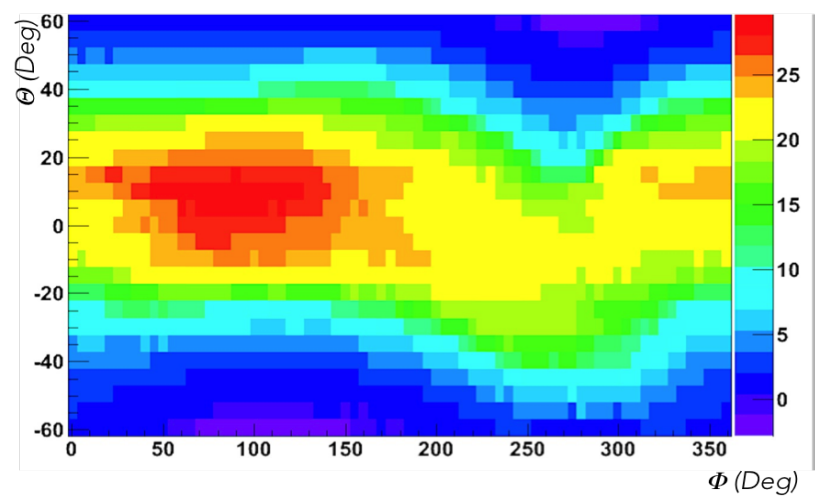

Fig. 7. Upper cutoff map for positively charged particles as a function of geographic latitude $(\theta)$ and longitude $(\varphi)$ 
negatively charged particle trajectories, as shown in Fig. 7 as a function of geographic latitude and longitude and in Fig. 8 as a function of time.

The upper cutoff shows remarkable differences between positive and negative particles (Fig. 8), with an anti-correlation with respect to the magnetic latitude. Infact, the cutoff of a positive particle is the same as for a negative particle arriving from the opposite direction in the conjugate position of field. This is not valid for the lower cutoff, where there is not any noticeable charge dependence for this cutoff, as expected since trapped tracjectories for positive and negative particles are the same, except that they are spanned in opposite longitudinal drifting direction.

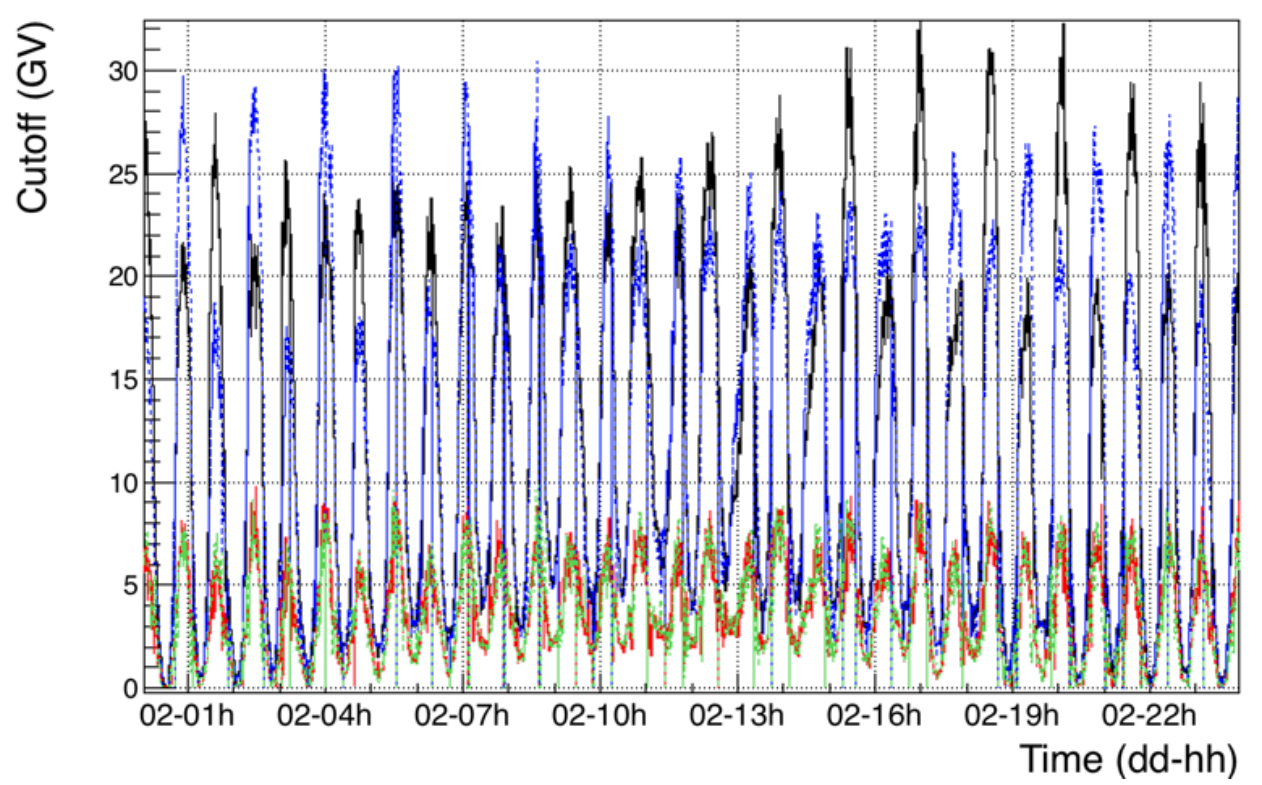

Fig. 8. Upper e lower cutoff for positive particles (black and red curves, respectively) as a function of time and for negative particles (blu and green curves, respectively) for July $2^{\text {nd }} 2011$.

By comparing the cutoffs obtained wih and without external field (fig. 9), it is clear that for a proper description of the geomagnetic field, the inclusion of the external

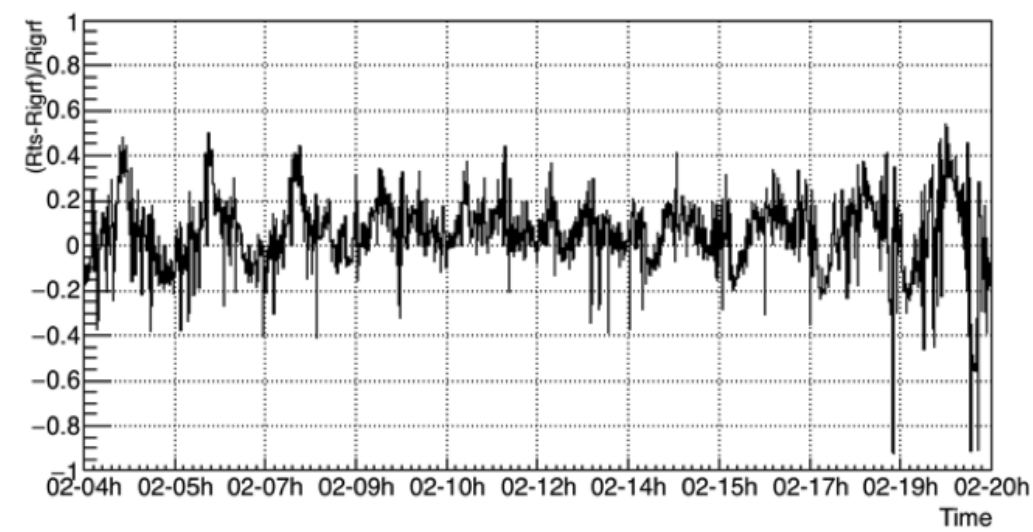

Fig. 9. Relative difference between the cutoff calculated with and without external field model, Tsyganenko '05 as a function of time. 
contribution is crucial, since the IGRF alone underestimates the cutoff at high latitudes and overestimate it at low ones, with a discrepancy up to $80 \%$ in polar regions.

\section{Conclusions}

In this work a method to calculate realistic time dependent geomagnetic cutoffs along the ISS orbit has been presented. The cutoffs were calculated in a $40^{\circ}$ cone around the pointing direction of the AMS02 apparatus, installed on the ISS for trajectories of positively and negatively charged particles. Two average cutoffs as a function of time (and therefore position) have been calculated: the upper cutoff, as the minimum rigidity where all trajectories are primary and the lower cutoff, as the maximum rigidity where all the trajectories are trapped. The region in between defines the penumbra region that turns out linearly scaling with the cutoff rigidity. The results show that even for low earth orbits the inclusion of the external field contribution is crucial for a proper description of the cutoffs.

\section{Aknowledgments}

This work has been supported by the Italian Space Agency under contracts ASI-INFN $\mathrm{I} / 002 / 13 / 0$ and $\mathrm{I} / 037 / 14 / 0$

\section{References}

[1] C. Stoermer, The PolarAurora,Oxford, 1955 and references therein.

[2] G. Lemaitre and M.S. Vailarta, Phys. Rev. 49 (1936) 719.

[3] C. C. IAGA Working Group V-MOD: Finlay, S. Maus, C. D. Beggan, and et al. International geomagnetic reference field: the eleventh generation. Geophysical Journal International, 183(3):1216-1230, 2010.

[4] N. A. Tsyganenko. A model of the near magnetosphere with a dawn-dusk asymmetry. Journal of Geophysical Research, 107(A8), 2002.

[5] Pfitzer, K.A., Olson, W.P. and Mogstad, T., A time dependent source driven magnetospheric magnetic field model, EOS 69 (1988) 426 [8] Olson, W.P. and Pfitzer, K.A., Magnetospheric magnetic field modeling, Annual Scienti fic Report, AFOSR Contract No. F44620-75-C-0033 (1977)

[6] Ostapenko, A.A., and Maltsev, Y.P., Relation of the magnetic field in the magnetosphere to the geomagnetic and solar wind activity, JGR 102 (1997) 1746717473

[7] Tsyganenko, N. A., and M. I. Sitnov (2005), Modeling the dynamics of the inner magnetosphere during strong geomagnetic storms, J. Geophys. Res., 110, A03208, doi: 10.1029/2004JA010798.

[8] M. Aguilar et al.Phys. Rev. Lett. 110, 141102 - Published 3 April 2013 
[9] D.F. Smart and M.A. Shea, Geomagnetic Cutoff Rigidity Calculations at 50-Year Intervals Between 1600 and 2000, 28th International Cosmic Ray Conference, 2003.

[10] http://www.magnetosphere.ru/iso/model/a2000.txt and ref in https://www.spenvis.oma.be/help/background/mag field/mag field.html\#ISO

[11] I.I. Alexeeva, Ya.I. Feldstein, Modeling of geomagnetic field during magnetic storms and comparison with observations, Journal of Atmospheric and Solar-Terrestrial Physics 63 (2001) 431-440

[12] Shea, M. A. and Smart, D. F., On the application of trajectory- derived cutoff rigidities to cosmic ray intensity variations, Acta Phys., 29, 533-537, 1970.

[13] Shea, M. A. and Smart, D. F., Estimating cosmic ray vertical cutoff rigidities as a function of the McIlwain L-parameter for different epochs of the geomagnetic field, Phys. Earth Planet. Int., 48, 200-205, 1986.

[14] D. Grandi et al., Trajectory reconstruction in the Earth Magnetosphere using TS05 model and evaluation of geomagnetic cutoff in AMS-02 data, ICRC2015-I/529, same proc.

[15] http://trend.aeronomie.be/trend4/unilib/home.shtml\#doc.

[16] E. Fiandrini et al., 2004, Leptons with E>200 MeV trapped in the Earth's radiation belts, 2002. 7pp. J. of Geo., Res.107,A6 10, 2002.

[17] E. Fiandrini et al., 2004, Leptons with E > $200 \mathrm{MeV}$ trapped near the South Atlantic Anomaly, 2003, 11pp. J. of Geo., Res.108, A11 1402, 2003.

[18] E. Fiandrini et al., 2004, Protons with kinetic energy E > $70 \mathrm{MeV}$ in the Earth's radiaion belts,12pp. J. of Geo., Res.109, A10214, 2004. 